\title{
Violation of the Wiedemann-Franz law for one-dimensional ultracold atomic gases
}

\author{
Michele Filippone, ${ }^{1}$ Frank Hekking, ${ }^{2,3}$ and Anna Minguzzi ${ }^{2,3}$ \\ ${ }^{1}$ Dahlem Center for Complex Quantum Systems and Institut für Theoretische Physik, Freie Universität Berlin, \\ Arnimallee 14, 14195 Berlin, Germany \\ ${ }^{2}$ Université Grenoble Alpes, LPMMC, F-38000 Grenoble, France \\ ${ }^{3}$ CNRS, LPMMC, F-38000 Grenoble, France
}

(Received 24 October 2014; published 5 January 2016)

\begin{abstract}
We study energy and particle transport for one-dimensional strongly interacting bosons through a ballistic single channel connecting two atomic reservoirs. We show the emergence of particle- and energy-current separation, leading to the violation of the Wiedemann-Franz law. As a consequence, we predict different time scales for the equilibration of temperature and particle imbalances between the reservoirs. Going beyond the linear spectrum approximation, we show the emergence of thermoelectric effects, which could be controlled by either tuning interactions or the temperature. Our results describe, in a unified picture, fermions in condensed-matter devices and bosons in ultracold atom setups. We conclude by discussing the effects of a controllable disorder.
\end{abstract}

DOI: 10.1103/PhysRevA.93.011602

The recent observations of mesoscopic transport in cold atomic gases [1] pave the way for the investigation of phasecoherent transport and thermoelectric effects with neutral bosonic atoms, where one compares heat and mass-rather than charge-transport. This stimulates theoretical studies [2] that focus on observables and probes that are different from-and complementary to- those studied for electronic devices.

A fundamental hallmark of electronic transport in metals is the Wiedemann-Franz (WF) law [3]. This law establishes that the ratio between thermal conductivity $\mathcal{K}$, electric conductivity $g$, and temperature $T$, known as the Lorenz number $L$, is a universal constant $L_{0}$ given by

$$
L \equiv \frac{\mathcal{K}}{g T}=\frac{\pi^{2}}{3}\left(\frac{k_{B}}{e}\right)^{2} \equiv L_{0} .
$$

The Drude [4] and the Fermi-liquid theory of transport [5,6] provide a microscopic interpretation of this law: low-energy quasiparticles carry both charge and energy. Deviations from the WF law (1) are then considered as a signature either of the breakdown of the quasiparticle character of low-energy excitations or of the presence of strongly energy-dependent quasiparticle scattering [7]. Indeed, deviations are expected for three-dimensional (3D) Fermi liquids [4,5], and have been observed in high- $T_{c}$ superconductors [8], close to phase transitions [9], and in quasi-one-dimensional strongly interacting channels [10]. Moreover, the nonlocality of quantum transport in mesoscopic systems requires generalization of the WF law to conductances instead of conductivities [11].

In one dimension (1D), interaction screening is much less effective, leading to the failure of the one-body picture. Low-energy collective modes emerge as a complicated superposition of the elementary constituents. The Fermi-liquid theory breaks down and must be replaced by an effective hydrodynamic approach, i.e., the Luttinger liquid (LL) theory [12]. The linearization of the spectrum close to the Fermi surface allows one to describe the low-energy excitations as a collection of noninteracting bosonic oscillators, characterized by the sound velocity $u$ and the interaction parameter $K$. The emergence of neutral collective modes, responsible for energy transport and distinct from the elementary constituents, carrying charge, leads to the violation of the WF law [13-16], i.e., the Lorenz number is different from $L_{0}$ in the presence of interactions.

While both interacting 1D bosons and fermions are similarly described by the LL theory, the Luttinger parameters describe strongly interacting $1 \mathrm{D}$ bosons with $K \geqslant 1[17,18]$, whereas $K \leqslant 1$ for repulsive fermions. Furthermore, reservoirs are interacting in the bosonic case, which is seldom the case for electronic devices due to strong screening of the Coulomb interaction. In view of the recent experimental advances with ultracold gases, it is therefore a timely issue to explore the fate of WF law for strongly interacting 1D bosons.

We focus on the two-terminal setup sketched in Fig. 1. A left $(\mathrm{L})$ and right $(\mathrm{R})$ reservoir are connected by a ballistic channel of length $d_{\mathrm{ch}}$. The interaction strength is assumed

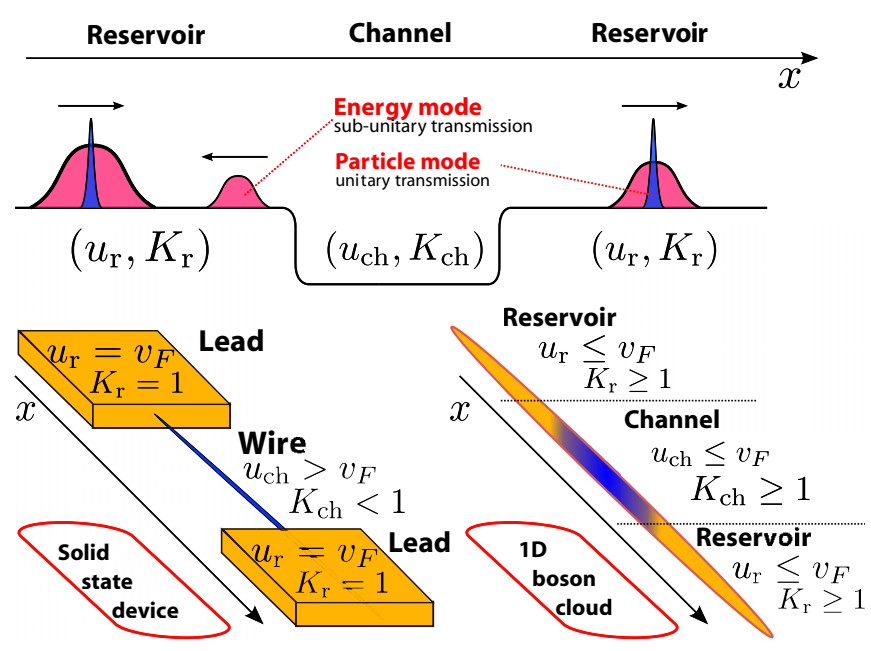

FIG. 1. Particle and energy transport are differently affected by spatial variations of the LL parameters $(u, K)$. In solid-state devices, this is observable in leads connected by a nanowire. In 1D bosonic clouds, the confining potential can be engineered in such a way as to connect two reservoirs by an atomic waveguide. A larger parameter region becomes accessible in which $K \neq 1$ not exclusively in the conducting channel. 
to be different in the channel and in the reservoirs, as could be engineered by adjusting the transverse confinement and hence the background density in each region. Our derivation of the thermoelectric coefficients, their correction to the Lorenz number, and their relation to the time-scale separation for the equilibration of reservoirs are contributions to the study of transport in interacting 1D systems.

Transport coefficients and model. Particle and energy currents, $J$ and $J_{\mathrm{E}}$, generated by a difference of chemical potential $\Delta \mu=\mu_{\mathrm{L}}-\mu_{\mathrm{R}}$ or temperature $\Delta T=T_{\mathrm{L}}-T_{\mathrm{R}}$ are given by the transport matrix [19]

$$
\left(\begin{array}{c}
J \\
J_{\mathrm{E}}
\end{array}\right)=g\left(\begin{array}{cc}
1 & s / T \\
s T & L+s^{2}
\end{array}\right)\left(\begin{array}{c}
\Delta \mu \\
T \Delta T
\end{array}\right) .
$$

Its off-diagonal elements, related to Peltier and Seebeck effects, depend on the thermopower $s$, a manifestation of Onsager relations [20]. An equivalent formulation of the transport matrix involves entropy instead of energy currents [1].

We want to obtain and discuss all of the elements of the transport matrix. We first derive the effective low-energy Hamiltonian governing the system depicted in Fig. 1. It is obtained by quantization of the classical equations of hydrodynamics in 1D [21]. These involve the continuity equation $\partial_{t} n+$ $\partial_{x}(n v)=0$ and Newton's law: $m n d_{t} v=-\partial_{x} P-n \partial_{x} V_{\text {ext }}$. $n(x, t)$ and $v(x, t)$ are fields describing the density and velocity of a gas submitted to the pressure $P$ and an external potential $V_{\text {ext }}$. The equations are linearized close to equilibrium: we assume $n(x, t)=n_{0}(x)+\delta n(x, t)$ and introduce a first-order displacement field $\vartheta$ such that $v(x, t)=\partial_{t} \vartheta(x, t)$. The chemical potential $\mu[n]$ is introduced such that $\partial_{x} P=n \partial_{x} \mu[n]$. It encodes the information about interactions among bosons. The linearized equations of motion (see Supplemental Material [22]) lead to the energy of the system, $\varepsilon=\int d x\left\{\frac{m n_{0}}{2}\left(\partial_{t} \vartheta\right)^{2}+\right.$ $\left.\left.\frac{1}{2} \frac{\delta \mu}{\delta n}\right|_{n_{0}}\left[\partial_{x}\left(n_{0} \vartheta\right)\right]^{2}\right\}$. Conjugate fields $\theta(x)=\pi n_{0}(x) \vartheta(x)$ and $\Pi(x)=m \partial_{t} \vartheta(x) / \pi \hbar$ are introduced, describing density and current fluctuations. The standard quantization procedure $\left[\theta(x), \Pi\left(x^{\prime}\right)\right]=i \delta\left(x-x^{\prime}\right)$, with $u(x) K(x)=\pi n_{0}(x) \hbar / m$ and $u(x) / K(x)=\left.\frac{1}{\pi \hbar} \frac{\delta \mu}{\delta n}\right|_{n_{0}}$, leads to the inhomogeneous LL model [23-25]

$$
\mathcal{H}_{0}=\frac{\hbar}{2 \pi} \int d x\left[u(x) K(x)(\pi \Pi)^{2}+\frac{u(x)}{K(x)}\left(\partial_{x} \theta\right)^{2}\right],
$$

to describe the setup in Fig. 1. The velocity and interaction parameters $u(x)$ and $K(x)$ both depend on the strength of the interactions between the bosons [17] and have different values, $\left(u_{\mathrm{ch}}, K_{\mathrm{ch}}\right)$ or $\left(u_{\mathrm{r}}, K_{\mathrm{r}}\right)$, if $x$ is in the channel region $x \in\left[-\frac{d_{\mathrm{ch}}}{2}, \frac{d_{\mathrm{ch}}}{2}\right]$ or not. $K$ and $u$ change at the connection between channel and reservoirs. The connection is typically smooth on scales of the order of the interparticle distance $\sim 1 / n_{0}$, but abrupt compared to the wavelength of the low-energy modes. This assumption allows one to neglect particle backscattering in Eq. (3) and still consider sharp variations of $u$ and $K$, yielding backscattering of energy modes. This different behavior of particle and energy transport leads to the violation of the WF law. In electronic systems, electrons do not interact in the reservoirs, implying $K_{\mathrm{r}}=1$, and interact repulsively in the channel, i.e., $K_{\mathrm{ch}}<1$. In the bosonic case, $K=1$ corresponds to the Tonks-Girardeau (TG) limit of infinite interactions. Away from this limit, $K$ assumes superunitary values both
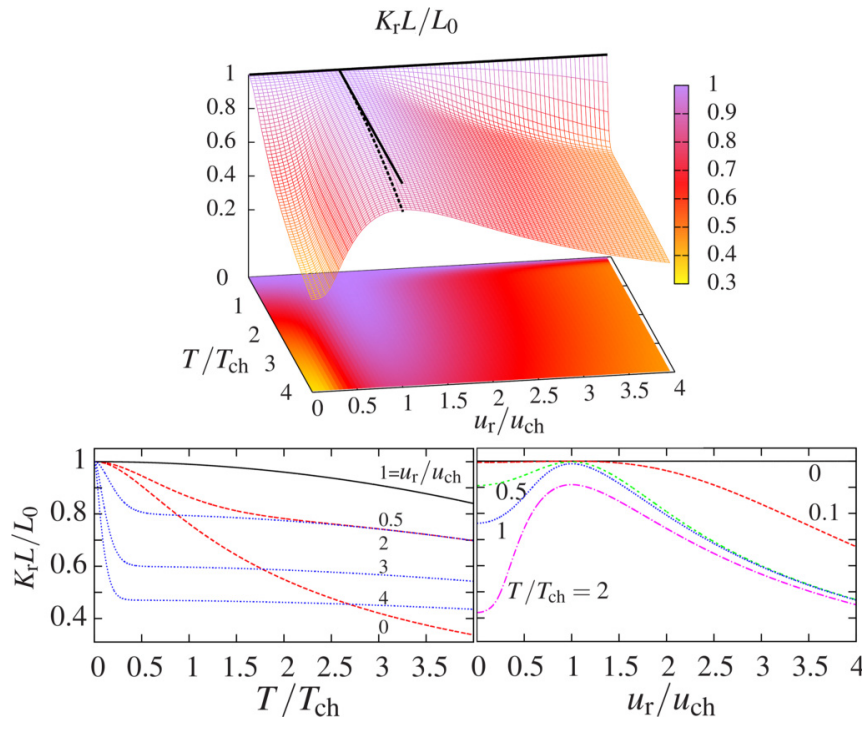

FIG. 2. Violation of the Wiedemann-Franz law. The Lorenz number (9) is plotted as a function of temperature and momentum mismatch of the energy modes at the channel-reservoir connections, quantified by the ratio $u_{\mathrm{r}} / u_{\mathrm{ch}}$. Increasing backscattering of energy modes at increasing temperatures, while particle transport is unaffected, implies a suppression of the Lorenz number. The WF law is violated also for $u_{\mathrm{r}}=u_{\mathrm{ch}}$ (compare solid with dashed black line) because of subleading thermoelectric effects. Calculations were carried out for the thermoelectric correction appearing in Eq. (9), with parameters in the reservoirs fulfilling $\pi^{2}\left(v_{F} / u_{\mathrm{r}}\right)^{2}\left(T_{\mathrm{ch}} / T_{F}\right)^{2} /$ $12 K_{\mathrm{r}}=10^{-2}$.

in the reservoirs and in the channel, with $K_{\mathrm{r}} \neq K_{\mathrm{ch}} \geqslant 1$, allowing one to explore completely new parameter regimes. The hydrodynamic approach used here is valid for strong to intermediate interactions, $K_{\mathrm{ch}, \mathrm{r}} \gtrsim 1$. In this regime, phase fluctuations are strong and effects related to the superfluid nature of the Bose gas can be ignored.

We define the energy density $h(x)$ in Eq. (3) as $\mathcal{H}_{0}=$ $\int d x h(x)$. The continuity equations $\partial_{x} J+\partial_{t} n=0$ and $\partial_{x} J_{\mathrm{E}}+\partial_{t} h=0$ for the particle and energy densities lead to the current operators,

$$
J=\pi u K \Pi, \quad J_{\mathrm{E}}=-\frac{\hbar u^{2}}{2}\left\{\Pi, \partial_{x} \theta\right\} .
$$

The linear conductance is readily obtained as $g=K_{\mathrm{r}} / h$; it is renormalized by interactions in the reservoirs [22,23,26]. We use the Landauer-Büttiker theory of coherent transport [27] to derive the thermal conductance $\mathcal{K}$. We diagonalize Eq. (3) with bosonic scattering states of energy $\omega$ and transmission amplitude $t_{\omega}$ through the channel [22]. The energy current reads $\left\langle J_{\mathrm{E}}\right\rangle=\frac{\hbar}{2 \pi} \int d \omega \omega\left|t_{\omega}\right|^{2}\left[n_{\mathrm{L}}(\omega)-n_{\mathrm{R}}(\omega)\right]$, in which $n_{\alpha}(\omega)$ is the Bose distribution of the scattering modes in the reservoirs. The thermal conductance $\mathcal{K}$ is defined in the $\Delta T \rightarrow 0$ limit of the energy current: $\left\langle J_{\mathrm{E}}\right\rangle=\mathcal{K} \Delta T$. The Lorenz number reads [13]

$$
L_{\mathrm{LS}}=\frac{\mathcal{K}}{g T}=\frac{L_{0}}{K_{\mathrm{r}}} \frac{6}{\pi^{2}} \int_{0}^{\infty} d x\left|t_{2 x / \hbar \beta}\right|^{2} \frac{x^{2}}{\sinh ^{2}(x)} .
$$


LS stands for the linear spectrum assumption implicit in Eq. (3). The transmission amplitudes $t_{\omega}$ depend only on frequencies $\omega$ and the ratio $u_{\mathrm{r}} / u_{\mathrm{ch}}$. The reason is that backscattering is due to the breaking of translational invariance at the channel-reservoir connection and hence to the momentum mismatch of incoming waves $\omega / u$ [22]. In the homogeneous situation $u_{\mathrm{r}}=u_{\mathrm{ch}}$, no change of eigenstates occurs along the cloud, so $t_{\omega}=1$. Yet, the Lorenz number is renormalized, $L_{\mathrm{LS}}=L_{0} / K_{\mathrm{r}}$, due to the renormalization of the linear conductance $g$ discussed above [14]. Interestingly, the WF law is satisfied in the Tonks-Girardeau limit of infinite interactions, when $K_{\mathrm{r}}=1$. For bosons with intermediate interactions $K_{\mathrm{r}}>$ 1 , we predict a regime where $L_{\mathrm{LS}}<L_{0}$ : the particle flow is enhanced with respect to heat flow. This has to be contrasted with the electronic case where $K_{\mathrm{r}}=1$ and no violation of the WF law is expected. In the inhomogeneous case $u_{\mathrm{r}} \neq u_{\mathrm{ch}}$, the transmission amplitudes $t_{\omega}$ become strongly energy dependent and control an additional, temperature-dependent deviation from the WF law. The Lorenz number $L_{\mathrm{LS}}$ acquires a strong dependency on $u_{\mathrm{r}} / u_{\mathrm{ch}}$ and $T / T_{\mathrm{ch}}$, with $T_{\mathrm{ch}}=\hbar u_{\mathrm{r}} / 2 d_{\mathrm{ch}} k_{B}$ being a characteristic temperature associated with the presence of the channel.

Thermopower. In the absence of chemical potential bias, the above approach yields no particle current for any temperature imbalance. Thermoelectric effects are absent, $s=0$. In order to recover these effects, we go beyond the quadratic Hamiltonian (3); within the hydrodynamic approach, its corrections read [22]

$$
\mathcal{H}_{1}=-\int d x\left[\frac{\pi \hbar^{2}}{8 m}\left\{\Pi,\left\{\Pi,\left(\partial_{x} \theta\right)\right\}\right\}+\left.\frac{\delta^{2} \mu}{\delta n^{2}}\right|_{n_{0}} \frac{\left(\partial_{x} \theta\right)^{3}}{6 \pi^{3}}\right] .
$$

They describe the interaction between energy modes and lead to a modified particle current operator,

$$
J=\pi u K \Pi+\frac{1}{m u^{2}} J_{\mathrm{E}}
$$

With the same accuracy, the operator $J_{\mathrm{E}}$ is unchanged. The second term in Eq. (7) couples mass and energy flows leading to thermoelectric effects. It is controlled by the inverse mass, associated with the deviation from the linear spectrum. Using Eq. (7), we find that the thermopower is proportional to the thermal conductance,

$$
s=\frac{1}{2}\left(\frac{v_{F}}{u_{\mathrm{r}}}\right)^{2} \frac{\mathcal{K}}{g E_{F}},
$$

where $E_{F}=m v_{F}^{2} / 2$ is the Fermi energy of the TG limit $K=1$ and $v_{F}=\pi \hbar n_{0} / m$ is the Fermi velocity. Our derivation predicts, in general, a nonlinear behavior of the thermopower as a function of temperature, an exquisite signature of interactions. An important consequence of $s \neq 0$ is the modification of the Lorenz number [see Eq. (2)],

$$
L=L_{\mathrm{LS}}\left[1-\frac{\pi^{2}}{12} \frac{L_{\mathrm{LS}}}{L_{0}}\left(\frac{T}{T_{F}}\right)^{2}\left(\frac{v_{F}}{u_{\mathrm{r}}}\right)^{4}\right],
$$

with $T_{F}=E_{F} / k_{B}$. The dependence of the Lorenz number on the temperature and velocity ratio $u_{\mathrm{r}} / u_{\mathrm{ch}}$ is illustrated in Fig. 2. Note that for finite temperatures $L \neq L_{\mathrm{LS}}$, even in the homogeneous case $u_{\mathrm{r}}=u_{\mathrm{ch}}$, generalizing the above result obtained for a linear spectrum. Interestingly, the Lorenz
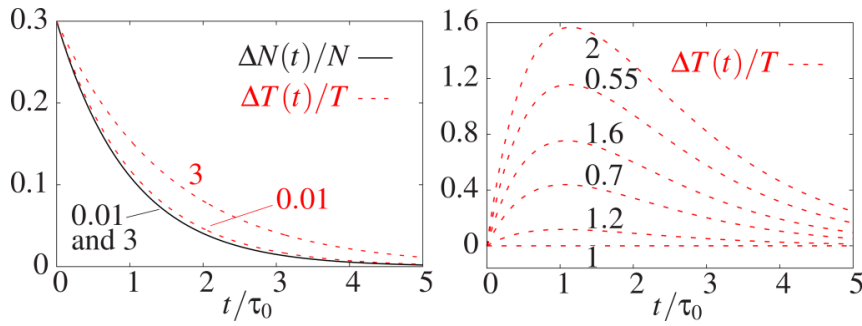

FIG. 3. Time evolution of particle (solid line) and temperature (dashed lines) imbalances. Left: Particle and temperature equilibrate with different time scales, as they are carried by different excitations. With increasing temperatures, the suppression of the Lorenz number slows down the decay of $\Delta T$. Numbers indicate different ratios of $T / T_{\mathrm{ch}}\left(K_{\mathrm{r}}=1.18, u_{\mathrm{r}}=0.8 v_{F}, T / T_{F}=0.2\right.$, and $\left.u_{\mathrm{r}} / u_{\mathrm{ch}}=2.5\right)$. Right: Thermoelectric effects lead to an evolution of the temperature imbalance, given an initial particle imbalance $[\Delta N(0) / N=0.3]$. Numbers indicate different ratios $u_{\mathrm{r}} / u_{\mathrm{ch}}$, showing that $\Delta(t) / T$ is tuned by interactions in the channel.

number behaves differently for $u_{\mathrm{r}}<u_{\mathrm{ch}}$ (the case of electrons in metallic devices; see Fig. 1) and $u_{\mathrm{r}}>u_{\mathrm{ch}}$ (realizable with ultracold atoms). This is a further manifestation of the wider range of possibilities offered by atomic setups. The Hamiltonian (6) implies a finite lifetime for the collective modes. Following Ref. [28], we estimate it for the relevant modes with wave vector $q \sim k_{B} T / \hbar u_{\text {ch }}$ and conclude that Eq. (9) holds for $T / T_{F}<\left(n_{0} d_{\mathrm{ch}}\right)^{-1 / 5}$, a realistic condition even if more stringent for long wires. The corrections to the conductance $g$ caused by finite lifetimes [29] can be neglected in this limit.

Time scales. Recent experiments [1] show the possibility to probe thermoelectric effects in the time evolution of the particle $\Delta N$ and temperature $\Delta T$ imbalances between reservoirs. They obey the differential equation

$$
\tau_{0} \partial_{t}\left(\begin{array}{c}
\Delta N \\
\Delta T
\end{array}\right)=-\left(\begin{array}{cc}
1 & -\kappa S \\
-\frac{S}{l \kappa} & \frac{L+S^{2}}{l}
\end{array}\right)\left(\begin{array}{c}
\Delta N \\
\Delta T
\end{array}\right) .
$$

$\tau_{0}=\kappa / g$ involves the compressibility of the reservoirs $\kappa=$ $\partial N /\left.\partial \mu\right|_{T}, l=C / \kappa T$, with specific heat $C=\partial E /\left.\partial T\right|_{N}$, and $S=s_{\mathrm{r}}-s$ is the dilatation coefficient with $\kappa s_{\mathrm{r}}=\partial N /\left.\partial T\right|_{\mu}$. Assuming $d_{\mathrm{r}}$ to be the size of the reservoirs, $\kappa=d_{\mathrm{r}} K_{\mathrm{r}} / \hbar \pi u_{\mathrm{r}}$ and $C=k_{B}^{2} d_{\mathrm{r}} T \pi / 3 \hbar u_{\mathrm{r}}[12]$.

We start by ignoring the thermoelectric effects $(S=0)$. In this limit, $\Delta N(t)=e^{-\frac{t}{\tau_{0}}} \Delta N(0)$, while $\Delta T(t)=$ $e^{-K_{\mathrm{r}} \frac{L_{\mathrm{LS}}}{L_{0}} \frac{t}{\tau_{0}}} \Delta T(0)$. Since, in general, $K_{\mathrm{r}} L_{\mathrm{LS}} / L_{0}<1$, we find that temperature imbalances relax with different time scales than particle imbalances; see left panel in Fig. 3. Taking into account the corrections to the linear spectrum given by Eq. (6), we derive [22] the dilatation coefficient $s_{\mathrm{r}}=C / m u_{\mathrm{r}}^{2} \kappa$, leading to

$$
S=\frac{L_{0}}{2 k_{B}}\left(\frac{v_{F}}{u_{\mathrm{r}}}\right)^{2} \frac{T}{T_{F}} \frac{1}{K_{\mathrm{r}}}\left[1-\frac{K_{\mathrm{r}} L_{\mathrm{LS}}}{L_{0}}\right] .
$$

As shown in Fig. 3, the presence of thermoelectric effects could be probed by preparing a particle imbalance and measuring the time evolution of the temperature imbalance, or vice versa. Remarkably, thermoelectric effects are extremely sensitive to 
the ratio $u_{\mathrm{r}} / u_{\mathrm{ch}}$, controlled by the variation of the interaction strength along the system. Thermoelectric response $S$ is equal to the difference between the thermopower $s$ of the channel and the dilatation coefficient $s_{r}$ of the reservoir. For $u_{\mathrm{r}}=u_{\mathrm{ch}}$, $s=s_{\mathrm{r}}$, hence $S=0$, leading to the absence of any response in Fig. 3.

Disorder effects. In ultracold atomic setups, tunable disorder can be added along the channel in a controllable way $[1,30]$. Here we discuss the consequences of disorder on bosonic transport. We identify two different regimes by comparing the disorder correlation length $l_{\mathrm{D}}$ to the interparticle distance $n_{0}^{-1}$.

(i) For $l_{\mathrm{D}} \lesssim n_{0}^{-1}$, particle backscattering affects mass transport. A renormalization-group (RG) analysis for $\delta$-correlated disorder allows one to determine its relevance at low energies in the presence of interactions [31]. Defining a dimensionless disorder strength $\mathcal{D}$ and a short-distance cutoff $a$, the scaling transformation $a \rightarrow a e^{l}$ determines the flow of the disorder strength,

$$
\mathcal{D}(l)=\mathcal{D} e^{\left(3-2 K_{\mathrm{ch}}\right) l} .
$$

For $K_{\mathrm{ch}}>3 / 2$, disorder is irrelevant and the cloud remains superfluid. Weak disorder yields a renormalization of the LL parameter $K_{\mathrm{ch}} \rightarrow K_{\mathrm{ch}}^{*}$ and channel-length $\left(d_{\mathrm{ch}}\right)$-dependent corrections to the WF law [15]. In the high-temperature limit $k_{B} T \gg \hbar u_{\mathrm{ch}} / d_{\mathrm{ch}}$, the conductance reads [32]

$$
g=\frac{K_{\mathrm{r}}}{h}\left[1-\mathcal{C} K_{\mathrm{r}} \frac{d_{\mathrm{ch}}}{a} \mathcal{D}\left(\frac{u_{\mathrm{ch}}}{T a}\right)^{2-2 K_{\mathrm{ch}}}\right]
$$

$\mathcal{C}$ is a nonuniversal factor depending on the UV regularization. The thermal conductance $\mathcal{K}$ will depend nonuniversally on the details of disorder. Increasing $d_{\mathrm{ch}}$, the second term in Eq. (13) becomes large and the conductance crosses over to an Ohm law $g \sim 1 / d_{\text {ch }}[15]$. In the low-temperature limit, $T \ll u_{\mathrm{ch}} / d_{\mathrm{ch}}$, and in the TG limit for the reservoirs $\left(K_{\mathrm{r}}=1\right)$ [32], the scaling of the $d_{\mathrm{ch}}$-dependent corrections to the conductance is derived by substituting $k_{B} T \Leftrightarrow \hbar u_{\mathrm{ch}} / d_{\mathrm{ch}}$ in Eq. (13) $[15,33]$.

Disorder becomes relevant in the RG sense for $K_{\mathrm{ch}}<3 / 2$. Below a localization temperature $T_{\text {loc }}$, the channel enters an insulating Bose glass phase [34]. The localization temperature tends to infinity in the TG limit, $K_{\mathrm{ch}}=1$. For $T>T_{\text {loc }}$, the channel remains superfluid and perturbative results in the disorder strength $\mathcal{D}$ apply.

(ii) For $l_{\mathrm{D}} \gg n_{0}^{-1}$, particle backscattering can be neglected, leaving mass transport unaffected, but long-wavelength energy modes propagate in a random medium [21]. The disorder is described by random fluctuations of the static density, $n_{0}(x) \rightarrow n_{0}(x)+\delta \rho(x)$, with $\left\langle\delta \rho(x) \delta \rho\left(x^{\prime}\right)\right\rangle=$ $\left\langle\delta \rho(x)^{2}\right\rangle \exp \left[-\left(x-x^{\prime}\right)^{2} / l_{\mathrm{D}}^{2}\right]$. Modes of energy $\omega$ acquire a mean free path $[13,21]$,

$$
\xi(\omega)=\frac{4 u_{\mathrm{ch}}^{6}}{\sqrt{\pi} l_{\mathrm{D}} \omega^{2} A^{2}\left\langle\delta \rho^{2}\right\rangle} e^{\omega^{2} l_{\mathrm{D}}^{2} / u_{\mathrm{ch}}^{2}} .
$$

We derive the factor $A$ in the strongly and weakly interacting limit by comparing the hydrodynamic approach to the exact solution of the Lieb-Liniger model [35]. Defining the dimensionless interaction strength $\gamma=m g / \hbar^{2} n_{0}$, we show that $A=$ $8 n_{0}(\pi \hbar / m)^{2} / \gamma$ for $\gamma \gg 1$ and $A=(\hbar / m)^{2} n_{0} \gamma$ for $\gamma \ll 1$ [22]. The mean free path diverges in the TG limit $\gamma \rightarrow \infty$ [36], which is understood using the mapping onto a free Fermi gas, not scattered by smooth disorder. Beyond the regime of validity of the LL picture, Eq. (14) should be matched at high energies with the free-particle behavior $\xi(\omega) \propto \omega^{2}$ [36], leading to a nonmonotonous dependence of the mean free path on energy. A nontrivial regime of particle and energy transport occurs when $\xi(\mu)>d_{\text {ch }}$ : particles are not localized within the sample; however, the energy modes are localized if their energy $\omega_{1}$ is sufficiently large (though still smaller than the chemical potential) such that $\xi\left(\omega_{1}\right)<d_{\text {ch. }}$. When $T>\omega_{1}$, this leads to a saturation of the thermal conductance, suppressing the Lorenz number. The energy $\omega_{1}$ has to be compared with the typical energy $\omega_{2} \sim v_{F} / \sqrt{n_{0}^{-1} d_{\text {ch }}}$ at which the scattering states spontaneously decay because of the interaction term given by Eq. (6). The condition $\omega_{2} / \omega_{1} \gg 1$ sets the validity of the scattering approach presented in the previous discussion. We recall that we found $\left\langle J_{\mathrm{E}}\right\rangle \sim \Delta T$. If $T>\omega_{2}$, high-energy modes are localized and interactions imply their decay into the low-energy ones, conducting heat. This down-energy conversion is responsible for an algebraic dependence of the energy current on the temperature imbalance [13,37],

$$
J_{\mathrm{E}} \sim \Delta T^{4 / 3} \text { if } \Delta T>\omega_{2} .
$$

This power law is a universal and exquisite effect of interaction between energy modes.

To conclude, we showed the violation of the WiedemannFranz law and thermoelectric effects in 1D cold atom clouds of strongly interacting bosons. They are signaled by the time evolution of particle or temperature imbalances. Our results generalize and extend previous results valid for fermions in electronic devices. In particular, we show that as a clear manifestation of interactions and the presence of reservoirs, the thermopower does not increase linearly with temperature, and we discuss the consequences on the Lorenz number. Interacting bosons in one dimension open new scenarios to explore transport in low-dimensional nanostructures [38] and probe various quantum phases, including many-body localization [34,39].

Acknowledgments. We thank D. Basko for discussions and M. Schneider for useful comments on the Rapid Communication. We acknowledge financial support from the Alexander von Humboldt Foundation, the Institut Universitaire de France, the ANR Project No. ANR-13-JS01-0005-01, and the HandyQ ERC StG Contract No. 258608.
[1] D. Stadler, S. Krinner, J. Meineke, J.-P. Brantut, and T. Esslinger, Nature (London) 491, 736 (2012); J.-P. Brantut, J. Meineke,
D. Stadler, S. Krinner, and T. Esslinger, Science 337, 1069 (2012); J.-P. Brantut, C. Grenier, J. Meineke, D. Stadler, 
S. Krinner, C. Kollath, T. Esslinger, and A. Georges, ibid. 342, 713 (2013); S. Krinner, D. Stadler, D. Husmann, J.-P. Brantut, and T. Esslinger, Nature 517, 64 (2015); D.Husmann, S.Uchino, S. Krinner, M. Lebrat, T. Giamarchi, T. Esslinger, and J.-P. Brantut, Science 350, 1498 (2015).

[2] A. J. Daley, P. Zoller, and B. Trauzettel, Phys. Rev. Lett. 100, 110404 (2008); I. Zapata and F. Sols, ibid. 102, 180405 (2009); D. B. Gutman, Y. Gefen, and A. D. Mirlin, Phys. Rev. B 85, 125102 (2012); A. Rancon, C. Chin, and K. Levin, New J. Phys. 16, 113072 (2014); D. P. Simpson, D. M. Gangardt, I. V. Lerner, and P. Krüger, Phys. Rev. Lett. 112, 100601 (2014); C. Nietner, G. Schaller, and T. Brandes, Phys. Rev. A 89, 013605 (2014); D. Papoular, L. Pitaevskii, and S. Stringari, Phys. Rev. Lett. 113, 170601 (2014).

[3] R. Franz and G. Wiedemann, Ann. Phys. 165, 497 (1853).

[4] N. W. Ashcroft and N. D. Mermin, Solid State Physics (Saunders, Philadelphia, 1976).

[5] A. A. Abrikosov, Fundamentals of the Theory of Metals (Elsevier Science, New York, 1988)

[6] P. Nozières and D. Pines, The Theory of Quantum Liquids, Vol. 6 (Perseus, Cambridge, MA, 1999).

[7] A. Casian, Phys. Rev. B 81, 155415 (2010).

[8] R. Hill, C. Proust, L. Taillefer, P. Fournier, and R. Greene, Nature (London) 414, 711 (2001).

[9] M. A. Tanatar, J. Paglione, C. Petrovic, and L. Taillefer, Science 316, 1320 (2007).

[10] N. Wakeham, A. F. Bangura, X. Xu, J.-F. Mercure, M. Greenblatt, and N. E. Hussey, Nat. Commun. 2, 396 (2011).

[11] H. Van Houten, L. Molenkamp, C. Beenakker, and C. Foxon, Semicond. Sci. Technol. 7, B215 (1992).

[12] F. D. M. Haldane, Phys. Rev. Lett. 47, 1840 (1981); J. Phys. C 14, 2585 (1981); T. Giamarchi, Quantum Physics in One Dimension (Oxford University Press, Oxford, 2004).

[13] R. Fazio, F. W. J. Hekking, and D. E. Khmelnitskii, Phys. Rev. Lett. 80, 5611 (1998).

[14] C. L. Kane and M. P. A. Fisher, Phys. Rev. Lett. 76, 3192 (1996).

[15] M.-R. Li and E. Orignac, Europhys. Lett. 60, 432 (2002).

[16] A. Garg, D. Rasch, E. Shimshoni, and A. Rosch, Phys. Rev. Lett. 103, 096402 (2009).

[17] M. Cazalilla, J. Phys. B 37, S1 (2004); M. A. Cazalilla, R. Citro, T. Giamarchi, E. Orignac, and M. Rigol, Rev. Mod. Phys. 83, 1405 (2011).

[18] M. Cominotti, D. Rossini, M. Rizzi, F. Hekking, and A. Minguzzi, Phys. Rev. Lett. 113, 025301 (2014).
[19] G. D. Mahan, Many Particle Physics (Springer, New York, 2000).

[20] L. Onsager, Phys. Rev. 37, 405 (1931).

[21] A. Gramada and M. E. Raikh, Phys. Rev. B 55, 1661 (1997); 55, 7673 (1997).

[22] See Supplemental Material at http://link.aps.org/supplemental/ 10.1103/PhysRevA.93.011602 for the linearized equations of motion.

[23] D. L. Maslov and M. Stone, Phys. Rev. B 52, R5539 (1995); I. Safi and H. J. Schulz, ibid. 52, R17040 (1995); V. V. Ponomarenko, ibid. 52, R8666 (1995).

[24] D. Petrov, D. M. Gangardt, and G. V. Shlyapnikov, J. Phys. IV 116, 5 (2004).

[25] R. Citro, S. De Palo, E. Orignac, P. Pedri, and M.-L. Chiofalo, New J. Phys. 10, 045011 (2008).

[26] S. Tarucha, T. Honda, and T. Saku, Solid State Commun. 94, 413 (1995)

[27] R. Landauer, Philos. Mag. 21, 863 (1970); M. Büttiker, Phys. Rev. Lett. 65, 2901 (1990).

[28] Z. Ristivojevic and K. A. Matveev, Phys. Rev. B 89, 180507 (2014).

[29] K. A. Matveev and A. V. Andreev, Phys. Rev. Lett. 107, 056402 (2011).

[30] J. Billy, V. Josse, Z. Zuo, A. Bernard, B. Hambrecht, P. Lugan, D. Clément, L. Sanchez-Palencia, P. Bouyer, and A. Aspect, Nature (London) 453, 891 (2008).

[31] T. Giamarchi and H. J. Schulz, Phys. Rev. B 37, 325 (1988); Z. Ristivojevic, A. Petković, P. Le Doussal, and T. Giamarchi, Phys. Rev. Lett. 109, 026402 (2012); Phys Rev. B 90, 125144 (2014).

[32] D. L. Maslov, Phys. Rev. B 52, R14368 (1995).

[33] M. Ogata and H. Fukuyama, Phys. Rev. Lett. 73, 468 (1994).

[34] I. Aleiner, B. Altshuler, and G. Shlyapnikov, Nat. Phys. 6, 900 (2010).

[35] E. H. Lieb and W. Liniger, Phys. Rev. 130, 1605 (1963).

[36] N. Bilas and N. Pavloff, Eur. Phys. J. D 40, 387 (2006).

[37] Y. Levinson, Solid State Commun. 36, 73 (1980); Nonequilibrium Phonons in Nonmetallic Crystals (Elsevier, New York, 1986).

[38] J. Lee, S. Eckel, F. Jendrezjewski, C. J. Lobb, G. K. Campbell, and W. T. Hill III, arXiv:1506.08413.

[39] V. P. Michal, I. L. Aleiner, B. L. Altshuler, and G. V. Shlyapnikov, arXiv:1502.00282. 